\title{
Assessment of Sleep Spindle Density among Genetically Positive Spinocerebellar Ataxias Types 1, 2, and 3 Patients
}

\author{
Doniparthi Venkata Seshagiri ${ }^{a}$ Ragasudha Botta $^{b}$ Arun Sasidharan ${ }^{c}$ \\ Pramod Kumar Pal $^{b}$ Sanjeev Jain ${ }^{d}$ Ravi Yadav $^{b}$ Bindu M. Kutty ${ }^{c}$ \\ a Department of Neurology, National Institute of Mental Health and Neuro Sciences (NIMHANS), Bengaluru, India; \\ ${ }^{b}$ Department of Clinical Neurosciences, National Institute of Mental Health and Neuro Sciences (NIMHANS), Bengaluru, \\ India; ' Department of Neurophysiology, National Institute of Mental Health and Neuro Sciences (NIMHANS), Bengaluru, \\ India; ${ }^{\mathrm{d}}$ Department of Psychiatry, National Institute of Mental Health and Neuro Sciences (NIMHANS), Bengaluru, India
}

\section{Keywords}

Spinocerebellar ataxia · Sleep spindles · NREM sleep ·

Neurodegeneration · Biomarker

\begin{abstract}
Objective: The effect of thalamic degeneration in patients with spinocerebellar ataxias (SCA) and sleep spindle (SS) abnormalities has not been studied so far, although there is a strong association between these disorders. This study was done to evaluate and compare the SS densities (SSDs) of genetically proven autosomal dominant SCA1, SCA2 and SCA3 patients with controls. Methods: Prospectively and genetically confirmed cases of SCA and controls were recruited. Patients were assessed clinically, were evaluated with sleep questionnaires and an overnight polysomnography was performed. SSDs were analyzed using neuroloop gain plugin of Polyman version 1.15 software. Results: Eighteen patients of SCA1 $(n=6), \operatorname{SCA} 2(n=5), \operatorname{SCA} 3(n=7)$ and 6 controls were
\end{abstract}

\section{KARGER}

() 2018 S. Karger AG, Basel

E-Mail karger@karger.com

www.karger.com/aon recruited in our study. The mean age of SCA1 patients was $39.2 \pm 5.4$, of SCA2 patients was $30.8 \pm 9.5$ and of SCA3 patients was $35.4 \pm 6.4$ years. The mean duration of illness in SCA1 was $4.7 \pm 1.7$ years, in SCA2 it was $4.3 \pm 4.4$ years and in SCA 3 it was $5 \pm 2.3$ years. The median SSD values (percentage loop gain) during stage 2 of non-rapid eye movement sleep were $16.9 \%$ in SCA1, $0 \%$ in SCA2, $1.2 \%$ in SCA3 and $59.5 \%$ in controls. There was a significant difference in SSD values in SCA2 $(p=0.04), \operatorname{SCA} 3(p=0.02)$ patients and controls. Conclusion: SSDs were significantly decreased in patients with SCA, which is a novel finding. This is likely due to the "thalamic switch" disruption, observed as reduced SSDs in SCA2 and SCA3. Sleep spindle deficits could act as one of the biomarkers of ongoing neurodegeneration in the thalamic circuitry of SCA patients.

(c) 2018 S. Karger AG, Basel
Dr. Ravi Yadav

Additional Professor, Department of Neurology

National Institute of Mental Health and Neurosciences (NIMHANS)

Hosur Road, Bangalore, Karnataka 560029 (India)

E-Mail docravi20@yahoo.com 


\section{Introduction}

Spinocerebellar ataxias (SCA) are a group of hereditary ataxias characterized by varying dysfunctions of the brain stem and cerebellum. They are caused by the expansion of Cytosine, Adenosine and Guanine (CAG) repeats. Patients with SCAs present with dysarthria, pyramidal signs, oculomotor and spinal cord symptoms in addition to cerebellar manifestations, gradual deterioration of bulbar functions, slow saccadic eye movements and in some patients, ophthalmoparesis or parkinsonism [1-4]. SCA3 or Machado Joseph syndrome is the most common SCA that is recognized worldwide [5]. These disorders have many non-motor symptoms, which include sleep disturbances like REM sleep behavior disorder and restless leg syndrome periodic limb movements, abnormal motor control during sleep, REM sleep without atonia, insomnia and fragmentation of sleep.

Among all SCAs, sleep disorders were most commonly seen in SCA3. Most common sleep disorders seen in SCA3 were REM sleep behavior disorder, restless leg syndrome, insomnia, excessive day time sleepiness, excessive fragmentary myoclonus and others [6-8].

Degeneration of the thalamus to a varying extent is present in both SCA2 and SCA3 [9]. Thalamus and cerebral cortex have a crucial role to play in the expression of the most significant behavioral and bioelectric events of non-rapid eye movement (NREM) sleep along with other structures like basal forebrain, cerebellum and caudal brain stem [10].

Sleep spindles are distinctive electroencephalography (EEG) oscillations originating during the NREM stage. Spindles are seen in the EEG as sinusoidal waves with frequency in the range of 11-16 Hz. Spindle generating neuronal circuits are present in the intrathalamic network of nucleus reticularis thalami and thalamocortical cells. These circuits said to have a multitude of functions like maintenance of sleep quality and arousal threshold, cellular plasticity, and memory consolidation [10]. GABA currents generate the spindles during development.

Analytical methods including visual and automated for SS densities (SSDs) are variable and there is no gold standard as of now $[11,12]$. It is known that the generation of sleep spindles affected by age, cognition and sleep related issues like sleep deprivation, wake-fullness state and many other non-sleep related factors like artefacts, anatomy and external neuronal drive, and electrode distance affects the spindle amplitudes. Neuro-loop analysis specifically measures only the sleep-related loop gain, independent of the amplitude of the rhythms [13-15].

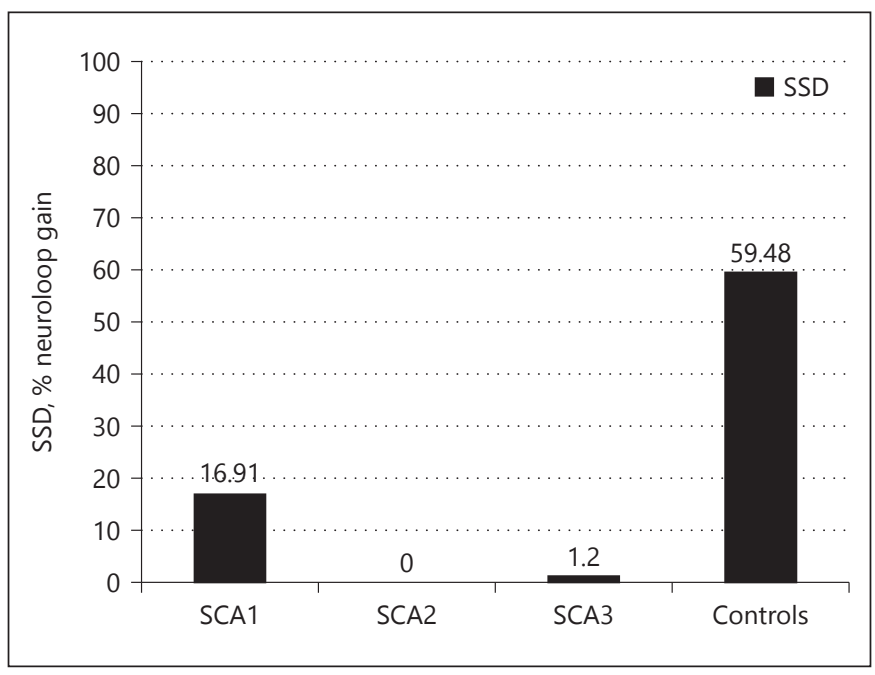

Fig. 1. Bar diagram showing the differences in sleep spindle densities (SSDs) in patients with Spinocerebellar ataxia (SCA) 1, 2, and 3 as compared to controls.

This study was conducted to study and compare the sleep spindle densities of genetically proven SCA1, SCA2 and SCA3 patients. The hypothesis of the study was that the thalamic degeneration in SCA leads to thalamic switch disruption, which would cause reduced sleep spindle density, as thalamo cortical integrity is essential for the formation of sleep spindles.

\section{Methodology}

Patients were recruited from the department of Neurology, National Institute of Mental Health and Neurosciences, Bangalore, after detailed clinical evaluation. Suspected cases of autosomal dominant SCA were tested for SCA1, SCA2 and SCA3 genetics. Eighteen genetically positive SCA1, SCA2 and SCA3 were recruited for the study. The severity of disease was assessed by the International Cooperative ataxia Rating Scale. Subjects who were on medications that affect sleep like antipsychotics, antidepressants and benzodiazapines were excluded. Age- and gender-matched controls were recruited. The presence of any movement disorder, dementia, chronic disease, alcoholism, other medical co-morbidities and medications that may affect sleep in the controls were excluded. All the patients and controls underwent overnight polysomnography after informed consent. Ethical clearance was obtained from the institutional review board.

Polysomnographic recordings were performed using the GalileoMizar-40video-EEG system(E.B.Neuro, Italy) which has 40 channels digitized with a resolution of 24 bits and sampling rate of $1,024 \mathrm{~Hz}$, a band pass filter of $0.1-500 \mathrm{~Hz}$ and no notch filters. Eleven monopolar paste-based silver disc electrodes (Grass Products, Natus Neurology, Warwick, USA) for EEG, 2 monopolar silver disc electrodes for electro-oculogram, and 2 silver discelectrodes for electro-myogram.EEG electrodes were positioned at F3, F4, C3, Cz, C4, P3, P4, T3, T4, O1 and $\mathrm{O} 2$ in accordance with the 10-20 international system for electrode 
Table 1. Clinical characteristics of patients with SCA $(n=18)$

\begin{tabular}{|c|c|c|c|}
\hline & $\operatorname{SCA} 1(n=6)$ & $\operatorname{SCA} 2(n=5)$ & $\operatorname{SCA} 3(n=7)$ \\
\hline Age, years, mean & $39.17 \pm 5.42$ & $30.80 \pm 9.45$ & $35.43 \pm 6.35$ \\
\hline Duration if illness, years & $4.67 \pm 1.63$ & $4.28 \pm 4.4$ & $5 \pm 2.3$ \\
\hline Severity of illness (ICARS) & $39.33 \pm 17.85$ & $29.4 \pm 14.32$ & $45.57 \pm 19.77$ \\
\hline CAG repeat length & $53.83 \pm 4.30(47-59)$ & $43.2 \pm 2.16(41-46)$ & $68.57 \pm 2.63(65-72)$ \\
\hline \multicolumn{4}{|l|}{ Clinical features } \\
\hline Ataxia & $100(6)$ & $100(5)$ & $100(7)$ \\
\hline Dysarthria & $100(6)$ & $100(5)$ & $85.7(6)$ \\
\hline Spasticity & $30(2)$ & $0(0)$ & $0(0)$ \\
\hline Dystonia & $0(0)$ & $0(0)$ & $0.1(1)$ \\
\hline Brisk reflexes & $33.3(2)$ & $20(1)$ & $28.5(2)$ \\
\hline Weakness/wasting & $0(0)$ & $0(0)$ & $0(0)$ \\
\hline Saccade abnormalities & $66.6(4)$ & $60(3)$ & $42(3)$ \\
\hline Pursuit abnormalities & $16.6(1)$ & $40(2)$ & $57.1(4)$ \\
\hline ESS & 1.8 & 2.4 & 4 \\
\hline RBDSQ & 0.5 & 0.2 & 1.6 \\
\hline HAM-A & 8.8 & 3.2 & 8.6 \\
\hline HAM-D & 6.7 & 1.4 & 4.7 \\
\hline PSQI & 4.5 & 3.6 & 4.6 \\
\hline
\end{tabular}

Values are $\%(n)$, mean $\pm \mathrm{SD}$ (range).

SCA, spinocerebellar ataxia; ICARS, International Cooperative Ataxia Rating Scale; CAG, Cytosine Adenine Guanine; ESS, Epworth Sleepiness Score; RBDSQ, rapid eye movement sleep behavior disorder sleep questionnaire; HAM-A, Hamilton Anxiety Rating Scale; HAM-D, Hamilton Depression Rating Scale; PSQI, Pittsburg Sleep Quality Index.

placement. Electrodes placed bilaterally on the mastoid area (M1 and M2) were used as reference during offline analysis. All electrodes were paste-based silver disc electrodes (Grass Products, Natus Neurology, Warwick, USA). Impedance for all electrodes was ensured to be between 5 and $10 \mathrm{~K} \Omega$ before starting acquisition.

Sleep scoring was done manually as per the American academy of sleep medicine guidelines, 2014, using Polyman software (Polyman-version 1.15, www.edfinfo.org). Sleep stage scoring was done by three different sleep experts who were blinded and these scores were used to prepare a consensus scoring.

EEG recordings in EDF format were used to analyze sleep spindle densities in Polyman software. The spindle densities were studied using the Neuroloop gain plugin of Polyman software. It was further analyzed using custom scripts written in MATLAB software (MATLAB-2013a, Mathworks, USA). For calculation of sleep spindle densities by neuroloop gain analysis or microcontinuity analysis, raw sleep EEG data from one of the central electrodes $(\mathrm{C} 3 / \mathrm{C} 4)$ referenced to opposite mastoid were used. Neuroloop-gain is a measure of micro-continuity of sleep-related delta and spindle oscillation, and is independent of the amplitude of EEG rhythms [15].

In this study, mean sleep spindle densities were analyzed automatically using Polyman version 1.15, neuroloop plugin. They were averaged across the NREM sleep using custom scripts written in MATLAB-2013a.

\section{Statistical Analysis}

Data expressed as median, mean, standard deviation for continuous variables and frequency, percentages for categorical variables was used. Kruskal-Wallis test was used to compare the SSDs of three SCA groups and controls. Pairwise comparison was done using post hoc Mann-Whitney $U$ test with Bonferroni correction. Significance was taken as $p<0.05$. Correlation between SSD and other variables was done using Spearman correlation. Partial correlation test was done to rule out the effect of confounding factors.

\section{Results}

Eighteen patients of SCA1 $(n=6)$, SCA2 $(n=5)$ and SCA3 $(n=7)$ were recruited for this study in addition to 6 controls. The mean age of SCA 1 patients was $39.17 \pm 5.42$, of SCA2 patients was $30.80 \pm 9.45$ and of SCA3 patients was $35.43 \pm 6.35$ years. The mean duration of illness in SCA1 was $4.67 \pm 1.63$ years, in SCA2 it was $4.28 \pm 4.4$ years, and in SCA3 it was $5 \pm 2.3$ years. Disease severity as measured by the International Cooperative ataxia Rating Scale was $39.33 \pm 17.85$ in SCA1, $29.4 \pm 14.32$ in SCA2 and $45.57 \pm$ 19.77 in SCA3 patients. The mean CAG repeat length was $53.83 \pm 4.30(47-59)$ in SCA1, $43.2 \pm 2.16(41-46)$ in SCA2 and $68.57 \pm 2.63(65-72)$ in SCA3 (Table 1).

Sleep parameters were recorded (Table 2 ). The median sleep spindle density values (percentage loop gain) during stage 2 of NREM sleep were $16.91 \%$ in SCA1, $0.0 \%$ in 
Table 2. Comparison of polysomnographic features in SCA1, 2 and 3

\begin{tabular}{lccc}
\hline & SCA1 & SCA2 & SCA3 \\
\hline Total sleep duration, $\mathrm{h}$ & $7.8 \pm 0.8$ & $7.6 \pm 0.5$ & $7.7 \pm 0.4$ \\
Sleep efficiency & $75.4 \pm 12.8$ & $79.7 \pm 15.9$ & $68.1 \pm 25.3$ \\
N1 percent & $25.2 \pm 12.1$ & $15.4 \pm 13.3$ & $21 \pm 13.6$ \\
N2 percent & $42.9 \pm 13.1$ & $61.1 \pm 9.7$ & $46.1 \pm 20.1$ \\
N3 percent & $21.5 \pm 10.6$ & $21.1 \pm 11.3$ & $27.6 \pm 17.3$ \\
REM percent & $10.4 \pm 7.0$ & $0.4 \pm 0.9$ & $8.3 \pm 12.1$ \\
N1 latency, min & $24.8 \pm 21.3$ & $17.1 \pm 11.2$ & $9 \pm 7.4$ \\
N2 latency, min & $37.6 \pm 17.5$ & $23.9 \pm 14.5$ & $16.6 \pm 10.8$ \\
\hline
\end{tabular}

Values are mean \pm SD.

$\mathrm{SCA}$, spinocerebellar ataxia; N1, N2, N3, stages of non rapid eye movement sleep; REM, rapid eye movement sleep.

SCA2, $1.2 \%$ in SCA3 and $59.48 \%$ in controls (Fig. 1). There was a significant difference in sleep spindle density values between SCA2 $(p=0.04)$, SCA3 $(p=0.02)$ patients and controls. However, SSD values between SCA1 and controls showed a decreasing trend $(p=0.052)$.

There was no significant difference in SSD values between SCA1, SCA2 and SCA3 patients (SCA1 vs. SCA2 $[p=0.26]$; SCA2 vs. SCA3 [ $p=1]$; SCA3 vs. SCA $1[p=$ $0.9])$. SSD values were not having any significant correlation to age $(p=0.44)$, gender $(p=0.45)$, disease severity score $(p=0.29)$, duration of illness $(p=0.18)$ or CAG repeat length $(p=0.46)$.

\section{Discussion}

Thalamic integrity plays a very important role in the proper functioning of various circuits like occulomotor, somatomotor and cerebellothalamocortical circuits. Various symptoms of SCA types 2 and 3 like gait, limb and truncal ataxia, frequent falls, dysarthria, saccadic disturbances and executive dysfunction attribute to the involvement of re-entrant circuits, which need thalamic integrity [9]. Silva et al. [16] evaluated 47 genetically proven cases of SCA 3 by polysomnographic studies and compared the results of these studies with those of the controls. They had reported significantly higher frequency of arousals from slow wave sleep to higher parasomnia complaints like confusional arousal/sleep terrors, higher REM sleep without atonia and more periodic limb movements of sleep. They showed that NREM parasomnias are significantly present in SCA3 [16]. However, our study was conducted only to study the SSD, hence Parasomnias could not be evaluated. Sensitive neuropsychological tests revealed deficits in abstraction, visual attention, verbal fluency, cognitive switching and in the shifting attentional behavior $[17,18]$. The clinical presentations of SCA2 and SCA3 were anatomically matched to the respective thalamic nuclei involvement by Rüb et al. [9] and showed the role of thalamus in various clinical manifestations. This study strengthened the role of thalamus in the pathogenesis of SCA2 and SCA3.

GABA receptors in the thalamus were shown to induce thalamic reticular nucleus neuron depolarization responsible for spindle burst generation. So, GABA currents play a pivot role in spindle generation. Previous imaging studies in Machado Joseph disease have revealed decreased GABAergic function in cortex, thalamus, striatum and cerebellum when compared to controls. The benzodiazapine receptor binding potential in cortex and thalamus was found to be decreased in these patients [19]. The decreased SSDs observed in our patients can be due to the GABAergic dysfunction in these patients caused due to thalamic degeneration.

Sleep studies in various trinucleotides had repeat disorders: Huntington's disease is CAG repeat disorder. Sleep pattern was found to be normal in patients of Huntington's disease when compared to controls and SSDs were significantly increased in these patients [20]. Piano et al. [21] had documented fragmented sleep, increased periodic limb movements, sleep apnea and daytime sleepiness in Huntington's disease. An example of a non-motor symptom is sleep disturbance with disrupted circadian rhythm and cognitive decline is commonly found in Huntington's disease. This might be due to the dysfunction of hypothalamus and limbic structures whose interconnections help in the maintenance of sleep, emotion and metabolism [22]. Thalamo-cortical circuitry dysfunction and thalamic atrophy were also reported in some studies and it was shown to covary with cognitive decline in Huntington's disease. Further studies are required to understand the correlation between thalamic volumes and sleep spindle density in these patients [23].

Friedreich's ataxia (GAA repeat disorder) patients were found to have increased prevalence of sleep disordered breathing and restless legs syndrome [24, 25]. Myotonic dystrophy is CTG trinucleotide repeat disorder. Several sleep disturbances like excessive day time sleepiness, sleep apneas, periodic leg movements during sleep, rapid eye movement sleep dysregulation, fatigue and hypersomnia were reported [26]. Myotonic dystrophy type 2 patients present with various sleep problems like sleep disordered breathing, restless legs syndrome, fatigue, in- 
somnia and day time sleepiness $[27,28]$. Studies on SSDs are lacking in these disorders.

In our study, sleep spindle densities were found to be decreased in SCA2 and SCA3 patients. This clearly shows the disruption of thalamocortical circuitry responsible for spindle generation.

SSDs did not correlate to age, duration of illness, severity of illness, CAG repeat length. This shows that the neurodegenerative process during certain point of time might involve the sleep spindle pacemaker, which is independent of the factors regulating the disease but the pathology itself. However, it has to be confirmed in a larger cohort.

Sleep spindle studies in other neurodegenerative diseases: sleep spindles are considered biomarkers of the ongoing neurodegeneration. Patients with Parkinson's disease (PD) have decreased sleep spindle densities and altered spindle characteristics when compared to controls [29]. It was found that patients with PD have a lower sleep spindle density compared to age- and gender-matched controls. According to Braak et al. [30], the neurodegenerative process in PD shows a progressive ascending course, which starts from the brain stem and spreads to other brain structures. At some point of time, the neurodegeneration may affect the SS generator of the thalamus, resulting in fewer or no spindles. Strikingly, it was found that medial thalamotomy abolishes spindle activity in N2 sleep systematically but that pallidothalamic tractotomy attenuates spindle activity only to a varying degree, with spindles reemerging after 3 months. It is therefore likely that the neurodegenerative involvement of prethalamic fibers from the brain stem may affect spindle activity to a certain degree [31]. Christensen et al. [29] has proposed sleep spindles as one of the biomarkers of ongoing neurodegeneration in PD. Substantial decrease in sleep spindles is seen in patients of progressive supranuclear palsy as compared to controls [32]. Alzheimer's disease patients have hippocampal alterations and reduction in sleep spindles. Sleep spindles were in turn correlated with the learning ability in these patients

\section{Limitations and Strengths of Our Study}

The limitation of our study was the small sample size and lack of neuropsychological scores because SSD has been associated with cognitive deficits. However, considering the rarity of these disorders, this is a satisfactory number.

This is probably the first study to evaluate the SSD abnormalities in patients with SCA. Our study shows a cortico-thalamic dysfunction in the form of reduced sleep spindle densities in SCA2 and SCA3 patients. This is a novel finding in SCA patients, but the pathophysiology could be due to GABA deficits as seen in other disorders.

\section{Conclusions}

Sleep spindle deficits could act as one of the biomarkers of ongoing neurodegeneration in the thalamic circuitry of SCA patients, but this observation needs to be validated in larger studies. The predilection of sleep spindle generator involvement in various neurodegenerative diseases can be highlighted. Further imaging studies may help to strengthen the relation between sleep spindle density values and cortico-thalamic involvement in SCA patients.

\section{Acknowledgment}

None.

\section{Disclosure Statement}

The authors have no conflicts of interest to declare.

\section{Funding Sources}

None.

\section{Author Contribution}

S.D. completed the data collection and R.B. did the primary data analysis and writing of the first draft. Genetic analysis was done at S.J.'s lab and results were discussed by him. The paper was reviewed and corrected with inputs from A.S., S.J., and P.K.P. R.Y. and B.M.K. have contributed equally in preparation of final manuscript.

\section{References}

\footnotetext{
1 Leggo J, Dalton A, Morrison PJ, Dodge A, Connarty M, Kotze MJ, Rubinsztein DC: Analysis of spinocerebellar ataxia types 1,2, 3, and 6, dentatorubral-pallidoluysian atrophy, and Friedreich's ataxia genes in spinocerebellar ataxia patients in the UK. J Med Genet 1997;34:982-985.

2 Subramony SH, Ashizawa T: Spinocerebellar ataxia type 1; in Pagon RA, Adam MP, Ardinger $\mathrm{HH}$, Wallace SE, Amemiya A, Bean LJ, Bird TD, Dolan CR, Fong CT, Smith RJ, Stephens K (eds): GeneReviews $\left({ }^{\circledR}\right)$. University of Washington, Seattle, WA, 1993.

3 Pulst SM: Spinocerebellar Ataxia Type 2, 2013.

4 Paulson H: Spinocerebellar Ataxia Type 3, 2011.
} 
5 Schöls L, Bauer P, Schmidt T, Schulte T, Riess O: Autosomal dominant cerebellar ataxias: clinical features, genetics, and pathogenesis. Lancet Neurol 2004;3:291-304.

6 Chi NF, Shiao GM, Ku HL, Soong BW: Sleep disruption in spinocerebellar ataxia type 3: a genetic and polysomnographic study. J Chin Med Assoc 2013;76:25-30.

7 dos Santos DF, Pedroso JL, Braga-Neto P, Silva GM, de Carvalho LB, Prado LB, Barsottini OG, do Prado GF: Excessive fragmentary myoclonus in Machado-Joseph disease. Sleep Med 2014;15:355-358.

8 Reimold M, Globas C, Gleichmann M, Schulze M, Gerloff C, Bares R, Machulla HJ, Bürk K: Spinocerebellar ataxia type 1,2, and 3 and restless legs syndrome: striatal dopamine D2 receptor status investigated by [11C]raclopride positron emission tomography. Mov Disord 2006;21:1667-1673.

$\checkmark 9$ Rüb U, Del Turco D, Del Tredici K, de Vos RA, Brunt ER, Reifenberger G, Seifried C, Schultz C, Auburger G, Braak H: Thalamic involvement in a spinocerebellar ataxia type 2 (SCA2) and a spinocerebellar ataxia type 3 (SCA3) patient, and its clinical relevance. Brain 2003;126:2257-2272.

10 de Andrés I, Garzón M, Reinoso-Suárez F: Functional anatomy of non-REM sleep. Front Neurol 2011;2:70.

11 Ujma PP, Gombos F, Genzel L, Konrad BN, Simor P, Steiger A, Dresler M, Bódizs R: A comparison of two sleep spindle detection methods based on all night averages: individually adjusted vs. fixed frequencies. Front Hum Neurosci 2015;9:52.

$\$ 12$ Ujma PP, Gombos F, Genzel L, Konrad BN, Simor P, Steiger A, Dresler M, Bódizs R: Corrigendum: a comparison of two sleep spindle detection methods based on all night averages: individually adjusted vs. fixed frequencies. Front Hum Neurosci 2015;9:415.

13 Clawson BC, Durkin J, Aton SJ: Form and function of sleep spindles across the lifespan. Neural Plast 2016;2016:6936381.
14 Kemp B: A proposal for computer-based sleep/ wake analysis. J Sleep Res 1993;2:179-185.

15 Kemp B, Zwinderman AH, Tuk B, Kamphuisen HA, Oberyé JJ: Analysis of a sleep-dependent neuronal feedback loop: the slow-wave microcontinuity of the EEG. IEEE Trans Biomed Eng 2000;47:1185-1194.

16 Silva GM, Pedroso JL, Dos Santos DF, BragaNeto P, Do Prado LB, De Carvalho LB, Barsottini OG, Do Prado GF: NREM-related parasomnias in Machado-Joseph disease: clini$\mathrm{cal}$ and polysomnographic evaluation. J Sleep Res 2016;25:11-15.

17 Maruff P, Tyler P, Burt T, Currie B, Burns C, Currie J: Cognitive deficits in Machado-Joseph disease. Ann Neurol 1996;40:421-427.

18 Le Pira F, Zappalà G, Saponara R, Domina E, Restivo D, Reggio E, Nicoletti A, Giuffrida S: Cognitive findings in spinocerebellar ataxia type 2: relationship to genetic and clinical variables. J Neurol Sci 2002;201:53-57.

19 Ishibashi M, Sakai T, Matsuishi T, Yonekura Y, Yamashita Y, Abe T, Ohnishi Y, Hayabuchi $\mathrm{N}$ : Decreased benzodiazepine receptor binding in Machado-Joseph disease. J Nucl Med 1998;39:1518-1520.

20 Emser W, Brenner M, Stober T, Schimrigk K: Changes in nocturnal sleep in Huntington's and Parkinson's disease. J Neurol 1988;235: 177-179.

21 Piano C, Losurdo A, Marca DG, Solito M, Calandra-Buonaura G, Provini F, Bentivoglio RA, Cortelli P: Polysomnographic findings and clinical correlates in Huntington disease: a Cross-Sectional Cohort Study. Sleep 2015; 38:1489-1495.

22 Petersén A, Gabery S: Hypothalamic and limbic system changes in Huntington's disease. J Huntingtons Dis 2012;1:5-16.

23 Kassubek J, Juengling FD, Ecker D, Landwehrmeyer GB: Thalamic atrophy in Huntington's disease co-varies with cognitive performance: a morphometric MRI analysis. Cereb Cortex 2005; 15:846-853.
24 Corben LA1, Ho M, Copland J, Tai G, Delatycki MB: Increased prevalence of sleep-disordered breathing in Friedreich ataxia. Neurology 2013;81:46-51.

25 Synofzik M, Godau J, Lindig T, Schöls L, Berg D: Restless legs and substantia nigra hypoechogenicity are common features in Friedreich's ataxia. Cerebellum 2011;10:9-13.

26 Kawai M: [Neuromuscular disease and sleep disturbance]. Rinsho Shinkeigaku 2014;54: 984-986.

27 Leonardis L, Blagus R, Dolenc Groselj L: Sleep and breathing disorders in myotonic dystrophy type 2. Acta Neurol Scand 2015;132:4248.

28 Romigi A, Placidi F, Albanese M, Izzi F, Liguori C, Mercuri NB, Marciani MG, Massa R, Silvestri G, Bianchi ML, Losurdo A, Della Marca G, St Louis EK, Lam EM, Shepard PW: Restless legs syndrome and daytime sleepiness are prominent in myotonic dystrophy type 2. Neurology 2014;83: 572.

29 Christensen JA, Nikolic M, Warby SC, Koch H, Zoetmulder M, Frandsen R, Moghadam KK, Sorensen HB, Mignot E, Jennum PJ: Sleep spindle alterations in patients with Parkinson's disease. Front Hum Neurosci 2015;9: 233.

30 Braak H, Del Tredici K, Rüb U, de Vos RA, Jansen Steur EN, Braak E: Staging of brain pathology related to sporadic Parkinson's disease. Neurobiol Aging 2003;24:197-211.

- 31 Roth C, Jeanmonod D, Magnin M, Morel A, Achermann P: Effects of medial thalamotomy and pallido-thalamic tractotomy on sleep and waking EEG in pain and Parkinsonian patients. Clin Neurophysiol 2000;111:12661275 .

32 Montplaisir J, Petit D, Décary A, Masson H, Bédard MA, Panisset M, Rémillard G, Gauthier S: Gauthier, sleep and quantitative EEG in patients with progressive supranuclear palsy. Neurology 1997;49:999-1003. 